\title{
Combining Ability and Gene Action Studies in Some Genetic Stocks of Tomato (Solanum lycopersicum L.)
}

\author{
Tanvi Raj ${ }^{1}$, Madan Lal Bhardwaj ${ }^{3}$, Saheb Pal $^{4 *}$ and Rajesh Kumar Dogra ${ }^{2}$ \\ ${ }^{1}$ Department of Vegetable Science, ${ }^{2}$ Department of Fruit Science, Dr. YS Parmar University of \\ Horticulture and Forestry, Nauni, Solan, Himachal Pradesh 173230, India \\ ${ }^{3}$ Regional Horticultural Research and Training Station, Dr. YS Parmar University of Horticulture \\ and Forestry, Jachh, Kangra, Himachal Pradesh 176202, India \\ ${ }^{4}$ Division of Vegetable Crops, ICAR- Indian Institute of Horticultural Research, Hessaraghatta, \\ Bengaluru, Karnataka 560089, India \\ *Corresponding author
}

A B S T R A C T

An experiment was conducted to find out the general and specific combining abilities of ten lines and two testers in tomato. Among the lines, EC-620410 was found good general

\section{Keywords}

Combining ability,

Gene action,

Tomato, GCA,

SCA, Line and

tester.

Article Info

Accepted:

04 September 2017

Available Online:

10 October 2017 combiner for days to $50 \%$ flowering and fruit shape index (P/E), BT-1-1 had maximum GCA for number of fruits per cluster, average fruit weight, fruit yield per plant, harvest duration, total soluble solids and ascorbic acid content and lesser severity of Alternaria, whereas, among the testers, FT-5 was good general combiner for most of the traits except for average fruit weight, number of locules per fruits and plant height. $F_{1}$ hybrid from cross combinations, EC-191535 $\times$ Solan Lalima was good in terms of earliness, had better fruit shape and fruit yield per plant. Hybrids of BT-1-1 $\times$ FT-5 were superior in terms of number of fruits per cluster and per plant, whereas, hybrids of BT-1-1 $\times$ Solan Lalima were found promising for average fruit weight. Predominance of non-additive gene action was recorded in almost all the traits under study except for total soluble solids and Alternaria blight severity, which showed dominant gene action. Therefore, most of the important yield and quality traits of tomato can be improved by heterosis breeding by selecting the parents having good specific combining ability for the target trait(s).

\section{Introduction}

Tomato (Solanum lycopersicum L.) is one of the most important vegetable crops in the world. India is the second largest producer of tomato after China with annual production of 18.73 million tonnes from an area of 0.88 million hectares (Anonymous, 2015). The national average productivity (21.2 tonnes/ha) is much lesser than that of world average (33.99 tonnes/ha) (FAOSTAT, 2014). The gap can be overcome through breeding of high yielding hybrids. The selection of parents for developing hybrids is most crucial decision that a breeder takes and direct selection, on the basis of per se performance doses not necessarily lead to desirable results (Allard, 1960). In this respect, the General Combining Ability (GCA) is used to designate the average performance of a line in several hybrid combinations and Specific Combining Ability (SCA) depicts certain 
combinations which perform relatively better or worse than expected on the basis of average performance (Sprague and Tatum, 1942). Thus combining ability is an effective tool to choose the parents for heterosis breeding in terms of the performance of their hybrids.

Line $\times$ tester analysis, proposed by Kempthorne (1957), is most commonly used for assessing combining ability. Further, it helps to evaluate relatively more number of germplasm lines in lesser time as compared to diallel and partial-diallel design. Therefore the objective of the present study was to estimate GCA as well as SCA of parents for several important horticultural traits to identify superior parental combinations in tomato and to find out the nature of gene action for important horticultural traits.

\section{Materials and Methods}

The investigation was conducted at the Experimental Research Farm, RHR\&TS, Jachh, Kangra, during Rabi, 2015. Twenty crosses were made in a line $\times$ tester fashion (Kempthorne, 1957) involving ten lines viz., EC-8910155, EC-191531, EC-191535, EC620410, EC-174913, EC-267727, EC-37239, LE-79-5, Yalabingo, BT-1-1 and two testers viz., Solan Lalima and FT-5, procured from different sources and maintained at Department of Vegetable Science, Dr YS Parmar University of Horticulture and Forestry, Nauni, Solan, Himachal Pradesh.

The twenty $F_{1}$ cross combinations and the parents were evaluated in a Randomized Complete Block Design (RCBD) with three replications during summer, 2016.

The row to row and plant to plant spacing was $90 \mathrm{~cm}$ and $30 \mathrm{~cm}$ respectively keeping twenty plants per plot $(2.7 \mathrm{~m} \times 2.0 \mathrm{~m})$ in each entry. Standard cultural practices for raising healthy crop of tomato were followed (Anonymous, 2013).

Data were recorded from five randomly selected plants (for plant characters) and 10 random fruits (for fruit characters) from each entry in each replication for days to 50 per cent flowering, number of fruits per cluster, number of marketable fruits per plant, average fruit weight $(\mathrm{g})$, fruit shape index (P/E ratio), number of locules per fruit, pericarp thickness $(\mathrm{mm})$, plant height $(\mathrm{cm})$, days to marketable maturity, harvest duration (days), Total Soluble Solids (TSS) $\left({ }^{0} \mathrm{~B}\right)$, ascorbic acid content (mg/100g), Alternaria blight severity and fruit yield per plant (g). TSS was measured with the help of a hand refractometer $\left(0-32^{0} \mathrm{~B}\right)$ of ERMA, Japan. Ascorbic acid content was estimated by adopting the method of Ranganna (1986) and by using the following formula:

$\mathrm{mg}$ of ascorbic acid per $100 \mathrm{~g}$ of fresh tissue=

Titre $\times$ Dye factor $\times$ Volume made up $\times 100$

Aliquot of extract $\times$ Volume of sample taken taken

Alternaria blight severity was recorded from 10 randomly selected leaves in each 5 random plants in each entry by adopting 0-5 scale of Shekhawat and Chakarvarti (1974), where, grade 0 denotes $0 \%$ leaf area infected and 5 implied more than $75.1 \%$ infected leaf area. The Percent Disease Index (PDI) was calculated according to the formula of McKinney (1923),

PDI of $\quad$ Sum of all disease ratings Alternaria $=$ Total number $\times$ Highest disease (\%) of ratings grade

The recorded data was subjected to analysis by MS-EXCEL, OPSTAT (available from CCS HAU, Hisar) and SPAR 2.0 (available from IASRI, New Delhi) packages. 
Int.J.Curr.Microbiol.App.Sci (2017) 6(10): 138-144

Table.1 Analysis of variance and the estimates of genetic components of variation for different traits in tomato

\begin{tabular}{|c|c|c|c|c|c|c|c|}
\hline Characters & Lines & Testers & $\begin{array}{l}\text { Line } \times \\
\text { testers }\end{array}$ & Error & $\sigma^{2} g$ & $\sigma^{2} s$ & $\sigma^{2} \mathrm{~g} / \sigma^{2} \mathrm{~s}$ \\
\hline Degrees of freedom & 9 & 1 & 9 & 62 & & & \\
\hline Days to 50 per cent flowering & $28.15^{*}$ & $4.82 *$ & $16.45^{*}$ & 0.55 & 0.004 & 5.251 & 0.001 \\
\hline Number of fruits per cluster & $0.81 *$ & $0.12 *$ & $1.68 *$ & 0.02 & -0.14 & 0.55 & -0.25 \\
\hline Number of marketable fruits per plant & $76.67 *$ & $41.00 *$ & $12.40 *$ & 0.25 & 5.16 & 4.01 & 1.29 \\
\hline Average fruit weight $(\mathrm{g})$ & $372.40^{*}$ & $174.75^{*}$ & $76.81^{*}$ & 0.55 & 21.86 & 25.46 & 0.86 \\
\hline Fruit yield per plant (g) & $174385.37 *$ & $60801.67 *$ & $58023.89 *$ & 458.64 & 6618.83 & 19192.79 & 0.34 \\
\hline Fruit yield per hectare (q) & $15694.68 *$ & $5472.15^{*}$ & $5222.15^{*}$ & 41.28 & 595.70 & 1727.35 & 0.34 \\
\hline Fruit shape index & $0.070 *$ & 0.0004 & $0.007 *$ & 0.0002 & 0.003 & 0.002 & 1.50 \\
\hline Number of locules per fruit & $0.92 *$ & $6.27 *$ & $1.03 *$ & 0.02 & 0.29 & 0.34 & 0.85 \\
\hline Pericarp thickness (mm) & $3.55 *$ & $0.27 *$ & $1.02 *$ & 0.01 & 0.10 & 0.33 & 0.30 \\
\hline Plant height $(\mathrm{cm})$ & $684.00 *$ & $16.66^{*}$ & $264.66^{*}$ & 1.75 & 9.52 & 87.50 & 0.11 \\
\hline Harvest duration & $3.42 *$ & 0.02 & 0.83 & 0.42 & 0.10 & 0.12 & 0.86 \\
\hline Days to marketable maturity & $24.04 *$ & $19.27 *$ & $29.49^{*}$ & 0.29 & -0.87 & 9.74 & -0.09 \\
\hline Total soluble solids $\left({ }^{\circ} \mathrm{B}\right)$ & $1.09 *$ & $4.22 *$ & $0.21 *$ & 0.01 & 0.27 & 0.07 & 4.00 \\
\hline Ascorbic acid content (mg/100g) & $49.81 *$ & $10.31 *$ & $5.73 *$ & 0.32 & 2.70 & 1.83 & 1.46 \\
\hline Alternaria blight severity (\%) & $162.62 *$ & $500.78 *$ & $57.95^{*}$ & 22.24 & 30.42 & 10.52 & 2.89 \\
\hline
\end{tabular}


Table.2 Estimates of general combining ability (GCA) effects of parents for different traits in tomato

\begin{tabular}{|c|c|c|c|c|c|c|c|c|c|c|c|c|c|c|c|}
\hline Parents & DTFPF & NOFPC & $\begin{array}{c}\text { NOMFP } \\
\mathbf{P}\end{array}$ & $\begin{array}{c}\mathbf{A F W} \\
(\mathrm{g})\end{array}$ & $\begin{array}{l}\text { FYPP } \\
(\mathrm{g})\end{array}$ & $\begin{array}{l}\text { FYPH } \\
\text { (q) }\end{array}$ & FSI & NOLPF & $\begin{array}{c}\text { PT } \\
(\mathbf{m m})\end{array}$ & $\begin{array}{c}\text { PH } \\
\text { (cm) }\end{array}$ & HD & DTMM & $\begin{array}{l}\text { TSS } \\
\left({ }^{\circ} \mathbf{B}\right)\end{array}$ & $\begin{array}{c}\mathbf{A C C} \\
(\mathrm{mg} / 100 \\
\mathrm{g})\end{array}$ & $\begin{array}{l}\text { ABS } \\
(\%)\end{array}$ \\
\hline \multicolumn{16}{|c|}{ Lines } \\
\hline BT-1-1 & $-1.72 *$ & $0.52 *$ & $0.76^{*}$ & $9.39 *$ & $249.50^{*}$ & $74.85^{*}$ & $-0.08^{*}$ & $-0.28 *$ & $0.49 *$ & $6.77 *$ & $0.85^{*}$ & $-1.77 *$ & $0.45^{*}$ & $4.24 * *$ & $-7.56^{*}$ \\
\hline EC-37239 & $2.28 *$ & $-0.25 *$ & $-4.50 *$ & $8.04 *$ & $-163.83^{*}$ & $-49.15^{*}$ & $-0.04 *$ & $-0.35 *$ & $1.16^{*}$ & $6.12 *$ & $0.52 *$ & 0.23 & -0.02 & $-1.00 * *$ & $6.89 *$ \\
\hline EC-191535 & $1.12 *$ & $-0.26 *$ & $-0.47 *$ & $6.68^{*}$ & $114.50^{*}$ & $34.35^{*}$ & $-0.12 *$ & $0.55^{*}$ & $0.26^{*}$ & $8.79 *$ & -0.15 & 0.07 & $0.32 * *$ & $2.64 * *$ & -1.10 \\
\hline EC-8910155 & $-1.22 *$ & $-0.18^{*}$ & $1.50^{*}$ & $-3.93^{*}$ & 14.50 & 4.35 & $-0.08^{*}$ & $-0.27 *$ & $0.10^{*}$ & $-14.33^{*}$ & 0.18 & $-0.77 *$ & $-0.72 * *$ & -0.13 & -3.11 \\
\hline EC-174913 & $1.28^{*}$ & $0.34 *$ & $-2.80^{*}$ & $-0.78^{*}$ & $-200.50^{*}$ & $-60.15^{*}$ & $-0.10^{*}$ & -0.08 & $0.26^{*}$ & $13.52 *$ & $-0.65 *$ & $-0.93 *$ & $-0.40 * *$ & $-3.49 * *$ & 2.45 \\
\hline EC-191531 & -0.55 & $-0.35^{*}$ & $-2.30^{*}$ & $4.97 *$ & $-75.50 *$ & $-22.65 *$ & $0.02^{*}$ & $-0.13 *$ & $0.30^{*}$ & $2.56^{*}$ & 0.35 & $3.23^{*}$ & $-0.57 * *$ & $-2.93 * *$ & 3.56 \\
\hline Yalabingo & $3.78^{*}$ & $0.42 *$ & -0.20 & $2.56^{*}$ & $59.50 *$ & $17.85^{*}$ & $-0.02 *$ & $-0.22 *$ & $-0.15^{*}$ & $6.78^{*}$ & $0.85^{*}$ & $0.73^{*}$ & 0.00 & $1.96^{* *}$ & -0.89 \\
\hline EC-267727 & 0.28 & $0.12 *$ & $0.56^{*}$ & $-2.53 *$ & -5.50 & -1.65 & $0.12 *$ & $-0.22 *$ & $0.19 *$ & $-3.93^{*}$ & $-1.32 *$ & $3.07 *$ & $0.37 * *$ & $-2.48 * *$ & -0.89 \\
\hline LE-79-5 & $-1.88^{*}$ & $0.19^{*}$ & $8.75^{*}$ & $-14.39 *$ & $237.83^{*}$ & $71.35^{*}$ & $0.13^{*}$ & $0.80 *$ & $-1.49^{*}$ & $-7.50 *$ & 0.35 & $-3.27 *$ & $0.37 * *$ & $3.54 * *$ & $-6.44 *$ \\
\hline EC-620410 & $-3.38^{*}$ & $-0.56^{*}$ & $-1.29 *$ & $-10.03^{*}$ & $-230.50^{*}$ & $-69.15^{*}$ & $0.18^{*}$ & $0.20 *$ & $-1.11^{*}$ & $-18.79 *$ & $-0.98 *$ & $-0.60 *$ & $0.21 * *$ & $-2.34 * *$ & $7.10^{*}$ \\
\hline \multicolumn{16}{|c|}{ Testers } \\
\hline FT-5 & $-0.28^{*}$ & $0.05^{*}$ & $0.83 *$ & $-1.71^{*}$ & $31.83^{*}$ & $9.55^{*}$ & -0.002 & $0.32 *$ & $0.07 *$ & $-0.53 *$ & 0.02 & $-0.57 *$ & $0.27 * *$ & $0.41 * *$ & $-2.89 *$ \\
\hline Solan Lalima & $0.28 *$ & $-0.05^{*}$ & $-0.83^{*}$ & $1.71 *$ & $-31.83^{*}$ & $-9.55^{*}$ & 0.002 & $-0.32 *$ & $-0.07^{*}$ & $0.53^{*}$ & -0.02 & $0.57 *$ & $-0.27 * *$ & $-0.41 * *$ & $2.89^{*}$ \\
\hline S.E. $\left(g_{\mathrm{i}}\right)$ Lines & 0.30 & 0.06 & 0.20 & 0.30 & 8.74 & 2.62 & 0.006 & 0.06 & 0.04 & 0.54 & 0.26 & 0.22 & 0.04 & 0.23 & 1.93 \\
\hline S.E. $\left(g_{j}\right)$ Testers & 0.14 & 0.03 & 0.09 & 0.14 & 3.91 & 1.17 & 0.003 & 0.03 & 0.02 & 0.24 & 0.12 & 0.10 & 0.02 & 0.10 & 0.86 \\
\hline S.E. $\left(g_{i}-g_{j}\right)$ Lines & 0.43 & 0.08 & 0.29 & 0.43 & 12.36 & 3.71 & 0.008 & 0.08 & 0.06 & 0.76 & 0.37 & 0.31 & 0.06 & 0.33 & 2.72 \\
\hline S.E. $\left(\mathrm{gi}-\mathrm{g}_{\mathrm{j}}\right)$ Testers & 0.19 & 0.04 & 0.13 & 0.19 & 5.53 & 1.66 & 0.004 & 0.04 & 0.03 & 0.34 & 0.17 & 0.14 & 0.03 & 0.15 & 1.22 \\
\hline C.D. .(0.05) $_{\left(\mathrm{g}_{\mathrm{i}}\right) \text { Lines }}$ & 0.59 & 0.11 & 0.40 & 0.59 & 17.14 & 5.14 & 0.011 & 0.11 & 0.08 & 1.06 & 0.52 & 0.43 & 0.08 & 0.45 & 3.77 \\
\hline C.D. ${ }_{(0.05)}\left(g_{j}\right)$ Testers & 0.27 & 0.05 & 0.18 & 0.27 & 7.66 & 2.30 & 0.005 & 0.05 & 0.04 & 0.47 & 0.23 & 0.19 & 0.04 & 0.20 & 1.69 \\
\hline C.D. ${ }_{(0.05)}\left(g_{i}-g_{j}\right)$ Lines & 0.84 & 0.16 & 0.57 & 0.84 & 24.23 & 7.27 & 0.016 & 0.16 & 0.11 & 1.50 & 0.73 & 0.61 & 0.11 & 0.64 & 5.34 \\
\hline $\begin{array}{l}\text { C.D.(0.05) }\left(g_{\mathrm{i}}-\mathrm{g}_{\mathrm{j}}\right) \\
\text { Testers }\end{array}$ & 0.38 & 0.07 & 0.25 & 0.38 & 10.84 & 3.25 & 0.007 & 0.07 & 0.05 & 0.67 & 0.33 & 0.27 & 0.05 & 0.29 & 2.39 \\
\hline
\end{tabular}

*Significant at $5 \%$ level of significance; ** Significant at $1 \%$ level of significance

Where,

\begin{tabular}{|c|c|c|c|c|c|c|}
\hline DTFPF & $=$ Days to 50 per cent flowering & $\begin{array}{l}\text { FYPH } \\
\text { (q) }\end{array}$ & $=$ Fruit yield per hectare $(\mathrm{q})$ & HD & $=$ & Harvest duration \\
\hline NOFPC & $=$ Number of fruits per cluster & FSI & $=$ Fruit shape index & DTMM & $=$ & Days to marketable maturity \\
\hline NOMFPP & $=$ Number of marketable fruits per plant & NOLPF & $=$ Number of locules per fruit & $\operatorname{TSS}\left({ }^{\circ} \mathrm{B}\right)$ & $=$ & Total soluble solids $\left({ }^{\circ} \mathrm{B}\right)$ \\
\hline AFW (g) & $=$ Average fruit weight $(\mathrm{g})$ & PT (mm) & $=$ Pericarp thickness $(\mathrm{mm})$ & $\begin{array}{l}\text { ACC } \quad(m g / 100 \\
\mathrm{g})\end{array}$ & $=$ & $\begin{array}{l}\begin{array}{l}\text { Ascorbic } \\
(\mathrm{mg} / 100 \mathrm{~g})\end{array} \\
(\text { acid }\end{array}$ \\
\hline FYPP (g) & $=$ Fruit yield per plant $(\mathrm{g})$ & PH (cm) & $=$ Plant height $(\mathrm{cm})$ & ABS $(\%)$ & $=$ & $\begin{array}{l}\text { Alternaria blight severity } \\
(\%)\end{array}$ \\
\hline
\end{tabular}


Table.3 Estimates of specific combining ability (SCA) effects of crosses for different traits in tomato

\begin{tabular}{|c|c|c|c|c|c|c|c|c|c|c|c|c|c|c|c|}
\hline \begin{tabular}{|lc} 
Crosses & Traits \\
\end{tabular} & DTFPF & NOFPC & NOMFPP & $\begin{array}{c}\text { AFW } \\
(g)\end{array}$ & $\begin{array}{c}\text { FYPP } \\
\text { (g) }\end{array}$ & $\begin{array}{c}\text { FYPH } \\
\text { (q) }\end{array}$ & FSI & NOLPF & $\begin{array}{c}\text { PT } \\
(\mathbf{m m})\end{array}$ & $\begin{array}{c}\mathbf{P H} \\
(\mathbf{c m})\end{array}$ & HD & DTMM & $\begin{array}{l}\text { TSS } \\
\left({ }^{\circ} \mathbf{B}\right)\end{array}$ & $\begin{array}{c}\mathrm{ACC} \\
(\mathbf{m g} / \mathbf{1 0 0} \mathbf{g})\end{array}$ & $\begin{array}{l}\text { ABS } \\
(\%)\end{array}$ \\
\hline BT-1-1 × FT-5 & $-1.72 *$ & $1.26^{*}$ & $3.01 *$ & $-5.67 *$ & $156.50^{*}$ & $46.95^{*}$ & $0.02 *$ & $-0.16^{*}$ & $0.50 *$ & $2.90 *$ & 0.48 & -0.27 & -0.07 & 0.25 & -0.44 \\
\hline BT-1-1 $\times$ Solan Lalima & $1.72^{*}$ & $-1.26^{*}$ & $-3.01 *$ & $5.67 *$ & $-156.50^{*}$ & $-46.95^{*}$ & $-0.02 *$ & $0.16^{*}$ & $-0.50^{*}$ & $-2.90 *$ & -0.48 & 0.27 & 0.07 & -0.25 & 0.44 \\
\hline EC-37239 $\times$ FT-5 & 0.62 & -0.08 & 0.54 & $-2.19 *$ & 16.50 & 4.95 & 0.01 & -0.06 & 0.09 & $12.00 *$ & -0.18 & $-1.60 *$ & $0.45^{* *}$ & -0.12 & -1.55 \\
\hline EC-37239 $\times$ Solan Lalima & -0.62 & 0.08 & -0.54 & $2.19 *$ & -16.50 & -4.95 & -0.01 & 0.06 & -0.09 & $-12.00 *$ & 0.18 & $1.60 *$ & $-0.45^{* *}$ & 0.12 & 1.55 \\
\hline EC-191535 × FT-5 & $3.12^{*}$ & $-0.33^{*}$ & $-1.76^{*}$ & $-1.88 *$ & $-158.50^{*}$ & $-47.55^{*}$ & $0.05^{*}$ & $0.44 *$ & $-0.21 *$ & $1.93 *$ & 0.48 & $3.57 *$ & $-0.16^{* *}$ & $-1.25^{* *}$ & 0.67 \\
\hline EC-191535 × Solan Lalima & $-3.12 *$ & $0.33^{*}$ & $1.76^{*}$ & $1.88^{*}$ & $158.50^{*}$ & $47.55^{*}$ & $-0.05^{*}$ & $-0.44 *$ & $0.21 *$ & $-1.93^{*}$ & -0.48 & $-3.57 *$ & $0.16^{* *}$ & $1.25 * *$ & -0.67 \\
\hline EC-8910155 × FT-5 & $1.12^{*}$ & -0.05 & 0.47 & $-2.24 *$ & -11.83 & -3.55 & -0.01 & $-0.57 *$ & $0.57 *$ & $-6.58^{*}$ & -0.18 & $-3.27 *$ & $-0.16^{* *}$ & $1.09 * *$ & 1.78 \\
\hline EC-8910155 × Solan Lalima & $-1.12 *$ & 0.05 & -0.47 & $2.24 *$ & 11.83 & 3.55 & 0.01 & $0.57 *$ & $-0.57 *$ & $6.58 *$ & 0.18 & $3.27 *$ & $0.16^{* *}$ & $-1.09 * *$ & -1.78 \\
\hline EC- $174913 \times$ FT-5 & $-1.05^{*}$ & $-0.33^{*}$ & $-0.63^{*}$ & $3.17 *$ & 16.50 & 4.95 & $0.03 *$ & $-0.59 *$ & $-0.71^{*}$ & $5.68^{*}$ & -0.02 & $-2.10^{*}$ & $-0.16^{* *}$ & $-1.38^{* *}$ & 0.67 \\
\hline EC- $174913 \times$ Solan Lalima & $1.05^{*}$ & $0.33^{*}$ & $0.63^{*}$ & $-3.17 *$ & -16.50 & -4.95 & $-0.03 *$ & $0.59 *$ & $0.71 *$ & $-5.68^{*}$ & 0.02 & $2.10^{*}$ & $0.16^{* *}$ & $1.38 * *$ & -0.67 \\
\hline EC-191531 × FT-5 & $-2.88 *$ & $-0.21 *$ & $-1.53 *$ & $4.92 *$ & $-48.50 *$ & $-14.55^{*}$ & $-0.06^{*}$ & -0.01 & $-0.18^{*}$ & $-2.46^{*}$ & 0.32 & 0.40 & -0.09 & $1.05^{* *}$ & 1.78 \\
\hline EC-191531 × Solan Lalima & $2.88^{*}$ & $0.21 *$ & $1.53 *$ & $-4.92 *$ & $48.50 *$ & 14.55 & $0.06^{*}$ & 0.01 & $0.18 *$ & $2.46^{*}$ & -0.32 & -0.40 & 0.09 & $-1.05^{* *}$ & -1.78 \\
\hline Yalabingo $\times$ FT-5 & -0.22 & -0.15 & $-1.56^{*}$ & $-1.00 *$ & $-133.50 *$ & $-40.05^{*}$ & $-0.03 *$ & $-0.16^{*}$ & $-0.25^{*}$ & $2.68^{*}$ & -0.52 & $-0.77 *$ & -0.01 & $1.00 * *$ & $6.22 *$ \\
\hline Yalabingo $\times$ Solan Lalima & 0.22 & 0.15 & $1.56^{*}$ & $1.00 *$ & $133.50 *$ & $40.05 *$ & $0.03 *$ & $0.16^{*}$ & $0.25 *$ & $-2.68^{*}$ & 0.52 & $0.77 *$ & 0.01 & $-1.00^{* * *}$ & $-6.22 *$ \\
\hline EC-267727 × FT-5 & -0.05 & $0.59 *$ & 0.41 & $-2.59 *$ & -11.83 & -3.55 & $0.02 *$ & 0.04 & $-0.28 *$ & $-11.15^{*}$ & 0.32 & 0.57 & 0.04 & 0.20 & -4.89 \\
\hline EC-267727 $\times$ Solan Lalima & 0.05 & $-0.59 *$ & -0.41 & $2.59 *$ & 11.83 & 3.55 & $-0.02 *$ & -0.04 & $0.28 *$ & $11.15^{*}$ & -0.32 & -0.57 & -0.04 & -0.20 & 4.89 \\
\hline LE-79-5 × FT-5 & 0.12 & $-0.18 *$ & $0.72 *$ & $3.81 *$ & $111.50 *$ & $33.45^{*}$ & $-0.02 *$ & $0.69 *$ & 0.01 & $-5.26^{*}$ & -0.35 & -0.10 & $0.13^{* *}$ & $-1.25^{* *}$ & -3.78 \\
\hline LE-79-5 × Solan Lalima & -0.12 & $0.18 *$ & $-0.72 *$ & $-3.81 *$ & $-111.50^{*}$ & $-33.45^{*}$ & $0.02 *$ & $-0.69^{*}$ & -0.01 & $5.26^{*}$ & 0.35 & 0.10 & $-0.13 * *$ & $1.25 * *$ & 3.78 \\
\hline EC- $620410 \times$ FT-5 & $0.95^{*}$ & $-0.53 *$ & 0.32 & $3.67 *$ & $63.17 *$ & $18.95^{*}$ & -0.01 & $0.36^{*}$ & $0.46^{*}$ & 0.28 & -0.35 & $3.57^{*}$ & 0.02 & 0.40 & -0.44 \\
\hline EC- $620410 \times$ Solan Lalima & $-0.95 *$ & $0.53 *$ & -0.32 & $-3.67 *$ & $-63.17 *$ & $-18.95 *$ & 0.01 & $-0.36^{*}$ & $-0.46^{*}$ & -0.28 & 0.35 & $-3.57 *$ & -0.02 & -0.40 & 0.44 \\
\hline S.E. $( \pm)$ & 0.43 & 0.08 & 0.29 & 0.43 & 12.36 & 3.71 & 0.01 & 0.08 & 0.06 & 0.76 & 0.37 & 0.31 & 0.06 & 0.33 & 2.72 \\
\hline \begin{tabular}{|l|} 
C.D. 0.05 \\
\end{tabular} & 0.84 & 0.16 & 0.57 & 0.84 & 24.33 & 7.27 & 0.02 & 0.16 & 0.11 & 1.50 & 0.73 & 0.61 & 0.11 & 0.64 & 5.34 \\
\hline
\end{tabular}

*Significant at $5 \%$ level of significance; ${ }^{* *}$ Significant at $1 \%$ level of significance

\begin{tabular}{|c|c|c|c|c|c|c|c|c|}
\hline DTFPF & $=$ & Days to 50 per cent flowering & $\begin{array}{l}\text { FYPH } \\
\text { (q) }\end{array}$ & $=$ & Fruit yield per hectare (q) & HD & $=$ & Harvest duration \\
\hline NOFPC & $=$ & Number of fruits per cluster & FSI & $=$ & Fruit shape index & DTMM & $=$ & $\begin{array}{l}\text { Days to marketable } \\
\text { maturity }\end{array}$ \\
\hline NOMFPP & $=$ & Number of marketable fruits per plant & NOLPF & $=$ & Number of locules per fruit & $\operatorname{TSS}\left({ }^{\circ} \mathrm{B}\right)$ & $=$ & Total soluble solids $\left({ }^{\circ} \mathrm{B}\right)$ \\
\hline AFW (g) & $=$ & Average fruit weight $(\mathrm{g})$ & PT (mm) & $=$ & Pericarp thickness (mm) & $\begin{array}{l}\text { ACC } \quad(m g / 100 \\
\mathrm{g})\end{array}$ & $=$ & $\begin{array}{l}\text { Ascorbic acid content } \\
(\mathrm{mg} / 100 \mathrm{~g})\end{array}$ \\
\hline FYPP (g) & $=$ & Fruit yield per plant (g) & $\mathrm{PH}(\mathrm{cm})$ & $=$ & Plant height $(\mathrm{cm})$ & ABS (\%) & $=$ & $\begin{array}{l}\text { Alternaria blight severity } \\
(\%)\end{array}$ \\
\hline
\end{tabular}




\section{Results and Discussion}

The analysis of variance and the estimates of different components of variance for combining ability effects on various characters of tomato have been presented on table 1 . The parent $v / s$ crosses were significant for all the traits under study. This indicates the effect of heterosis for all the traits. The variances for combining ability were highly significant for line, tester and line $\times$ tester. For all the characters under study, both GCA and SCA showed wide range of variation.

From the estimates of GCA as presented in table 2, none of the parents were found to be a good general combiner for all the characters. Among the lines, EC-620410 was found good general combiner for days to $50 \%$ flowering and fruit shape index (P/E), BT-1-1 had maximum GCA for number of fruits per cluster, average fruit weight, fruit yield per plant, harvest duration, total soluble solids and ascorbic acid content and least PDI of Alternaria. LE-79-5 was best general combiner with respect to days to marketable maturity and number of fruits per plant, whereas, EC-37239 was best general combiner for pericarp thickness and number of locules per fruit and the line, EC-174913 was best general combiner for plant height. In case of testers, FT-5 was good general combiner for most of the traits except for average fruit weight, number of locules per fruits and plant height, in which Solan Lalima was better. With respect to harvest duration, both the testers had nonsignificant general combining ability. The variance ratio for number of fruits per cluster and days to marketable maturity was estimated with negative values. Therefore this could be considered indifferent from zero.

The best hybrid combinations that recorded desirable values of SCA, as presented in the table 3, implied that these parental combinations will combine well and produce superior hybrids with respect to the particular traits. EC-191535 $\times$ Solan Lalima was best for improving traits viz., days to 50 percent flowering, fruit shape index (P/E ratio) and fruit yield per plant. For number of fruits per cluster and number of fruits per plant, BT-1-1 $\times$ FT-5 was found best and for average fruit weight, BT-1-1 $\times$ Solan Lalima was best. The crosses LE-79-5 $\times$ Solan Lalima and EC-174913 $\times$ Solan Lalima was best for number of locules per fruits and pericarp thickness as well as ascorbic acid content, respectively. EC-37239 × FT-5 was best with respect to total soluble solids content and plant height. The resultant of the combination, Yalabingo $\times$ Solan Lalima had least severity of Alternaria. No cross combinations were found significant for harvest duration. Similar general and specific combiners for different traits of tomato were earlier identified by Kumar et al., (2015) while working with ten lines and four testers in tomato.

The estimates of $\sigma^{2}$ SCA were higher in magnitude as compared to $\sigma^{2}$ GCA for most of the traits except for TSS and Alternaria blight severity, indicating the predominance of nonadditive gene action for those traits. Similar findings were also reported by Kapur et al., (2013) for days to 50 per cent flowering and harvest duration; by Kumari and Sharma (2012) for number of fruits per cluster and fruit yield per plant; by Saeed et al., (2014) for number of marketable fruits per plant; by Kumar et al., (2015) for average fruit weight, number of locules per fruit and pericarp thickness; by Saleem et al., (2013) for plant height and days to marketable maturity; by Kumar et al., (2013) for ascorbic acid content. Therefore, for improving these traits, heterosis breeding will be most practical.

Our result contradicted with the finding of Kumar et al., (2015), who reported non-additive gene action for total soluble solids content. Moreover, our results indicated dominant gene action for Alternaria resistance. Therefore in order to develop a new variety with early blight resistance and to improve TSS, breeder has to adopt selection as a breeding method. 


\section{Acknowledgement}

The authors are thankful to all the staffs of Regional Horticultural Research and Training Station, Dr. YS Parmar University of Horticulture and Forestry, Jachh, Kangra, Himachal Pradesh, India for providing all the facilities during the research trials.

\section{References}

Allard, R. W., 1960. Principles of Plant Breeding. John Wiley and Sons Inc. New York. Pp. 270-271.

Anonymous, 2013. Package of Practices for Vegetable Crops. Directorate of Extension Education, Dr. YS Parmar University of Horticulture and Forestry, Nauni, Solan. pp. 202.

Anonymous, 2015. Handbook of Indian Horticulture Database, NHB, Gurgaon. Department of Agriculture and Cooperation, Ministry of Agriculture, Government of India, New Delhi. pp. 48.

FAOSTAT, 2014. Available at http://www.fao.org/faostat/en/\#data. Date accessed: 09/08/2017.

Kapur, P., Vidyasagar and Chadha, S. 2013. Combining ability and gene action studies over environments in bacterial wilt resistant tomato genotypes. Vegetable Science 40(2): 164-168.

Kempthorne, O., 1957. An introduction to Genetic Statistics, John Wiley and Sons Inc. New York. pp. 458-471.

Kumar, R., Srivastava, K., Singh, R. K. and Kumar, V. 2013. Heterosis and quality attributes in tomato (Lycopersicon esculentum Mill.). Vegetos. 56(1): 101106.
Kumar, V., Jindal, S. K. and Dhaliwal, M. S. 2015. Combining ability studies in tomato (Solanum lycopersicum L.). Agriculture Research Journal. 52(2): 121-125.

Kumari, S., and Sharma, M. 2012. Line $\times$ tester analysis to study combining ability effects in tomato (Solanum lycopersicum L.). Vegetable Science. 39(1): 65-69.

McKinney, H.H., 1923. Influence of soil temperature and moisture on infection of wheat seedlings by Helminthosporium sativum. Journal of Agricultural Research. 26: $195-217$

Ranganna, S., 1986. Hand book of analysis and quality control for fruit and vegetable production. Tata McGraw Hill Publishing Company Limited, New Delhi. pp. 105106.

Saeed, A., Hasan, N., Shakeel, A., Saleem, M. F., Khan, N. H., Ziaf, K., Khan, R. A. M. and Saeed, N. 2014. Genetic analysis to find suitable parents for development of tomato hybrids. Life Science Journal. 11(12): 30-35.

Saleem, M. Y., Asghar, M., Iqbal, Q., Rehman, A. and Akram, M. 2013. Diallel analysis of yield and some yield components in tomato (Solanum lycopersicum L.). Pakistan Journal of Botany. 45(4): 12471250.

Shekhawat, P. S., and Chakarvati, B. P. 1974. Factor affecting development of bacterial leaf spot of chillies caused by Xanthomonas vesicatoria. Indian Phytopathology. 29: 393-397.

Sprague, G., and Tatum, A. 1942. General v/s specific combining ability in single crosses of corn. Journal of American Society of Agronomy. 34: 923-932.

\section{How to cite this article:}

Tanvi Raj, Madan Lal Bhardwaj, Saheb Pal and Rajesh Kumar Dogra. 2017. Combining Ability and Gene Action Studies in Some Genetic Stocks of Tomato (Solanum lycopersicum L.). Int.J.Curr.Microbiol.App.Sci. 6(10): 138-144. doi: https://doi.org/10.20546/ijcmas.2017.610.017 